\title{
Material educativo computacional (MEC) una herramienta didáctica para enseñar carbohidratos
}

\author{
Material educational computacional (mec) a didactic tool TO TEACH (SHOW) \\ CARBOHYDRATES
}

Yuly Constanza Bustos Contreras y Jimena Sandoval Sarrias.

Universidad Pedagógica Nacional yulyconstanzabc@yahoo.com;

ximena9090@gmail.com

\section{Resumen}

Dentro de la integración de las ciencias con la tecnología y el proceso educativo, el presente artículo muestra una propuesta que se basa en el diseño e implementación de un MEC como herramienta didáctica para la enseñanza de carbohidratos en la educación media, concepto que pocas veces se aborda a pesar de hacer parte de los contenidos básicos de ciencias naturales incluidos dentro de la propuesta de lineamientos curriculares (MEN, 1998). En este trabajo se evidencia, la importancia de innovar con herramientas visuales el trabajo dentro del aula, facilitando la labor del docente.

\section{Palabras claves}

TICs, MEC, carbohidratos, herramienta didáctica.

\section{Abstrac}

Within the integration of science with technology and educational process, this paper presents a proposal that is based on the design and implementation of MEC as a teaching tool for teaching middle school in carbohydrates, a concept that few sometimes deals despite being part of the basic natural sciences included in the proposed curriculum guidelines (MEN, 1998). This work shows the importance of innovations with visual tools work within the classroom, facilitating the work of teachers.

\section{Keywords}

TICS, MEC, carbohydrates, didactic tool.

\section{Introducción}

La Química como disciplina del saber, ha sido modificada por la introducción de herramientas de comunicación conocidas como TICs Tecnologías de la Información y la Comunicación cuya característica principal es la interconexión mundial a través de la red. Los sistemas multimedia, flexibles y asociados a la idea de interacción, comienzan a ser utilizados en la didáctica de la química, llegándose a hablar incluso de un cambio en la enseñanza de la química, catalizado por la tecnología multimedia (Jones y Smith, 1993).

En la última década del siglo XX se evidencia su inclusión masiva en procesos de formación en los distintos niveles educativos convirtiéndose en una oportunidad y un 
reto en la didáctica de las ciencias, que de acuerdo a las investigaciones ejecutadas desde entonces pone en manifiesto tres distintas maneras de incluirlas en los espacios escolares, primero como objeto de aprendizaje por que permite que los alumnos se familiaricen con el material computacional y adquieran las competencias necesarias para hacer del mismo un instrumento útil a lo largo de los estudios, en el mundo del trabajo o en la formación continua cuando sean adultos; segundo se consideran que las tecnologías son utilizadas como un medio de aprendizaje cuando es una herramienta al servicio de la formación a distancia, no presencial y del auto aprendizaje o son ejercicios de repetición, cursos en línea a través de Internet, de videoconferencia, programas de simulación o de ejercicios, etc; pero donde las nuevas tecnologías encuentran su verdadero sitio en la enseñanza es como apoyo al aprendizaje. Las tecnologías así entendidas se hayan pedagógicamente integradas en el proceso de aprendizaje, tienen su sitio en el aula, responden a unas necesidades de formación más proactivas y son empleadas de forma cotidiana. La integración didáctica de las tecnologías difiere de la formación en las tecnologías y se enmarca en una perspectiva de formación continua y de evolución personal y profesional como un saber aprender (Gómez, 2004).

De esta manera la presente propuesta consiste principalmente en la elaboración e implementación del MEC como herramienta de apoyo en la labor del docente para el aprendizaje del concepto asociados a los carbohidratos, generando un espacio alternativo donde el estudiante puede encontrar la información enmarcada dentro de un ambiente computacional, que produce en él la intencionalidad positiva para la exploración, el dinamismo y el descubrimiento para el abordaje de temáticas nuevas.

\section{Desarrollo}

El diseño curricular en ciencias involucra no solo lo respectivo a los planes de estudio, también se consideran las estrategias de construcción y criterios de evaluación (Arnaz, 1995), posibilitando el diseño de estrategias de enseñanza de carácter interdisciplinar en el que se pueden incluir herramientas tecnológicas como Ios MECs, para facilitar el aprendizaje significativo por medio de la promoción, la integración y el desarrollo de habilidades intelectuales como el razonamiento, la resolución de problemas, la creatividad, la imaginación fortaleciendo la capacidad de búsqueda y mejorando la motivación aprender a aprender (MEN, 1998).

Los MEC para algunos autores como Galvis (Galvis, 1996) son la denominación otorgada a las diferentes aplicaciones informáticas cuyo objetivo terminal es apoyar el aprendizaje. Se caracterizan porque es el estudiante quien controla el ritmo de aprendizaje, la cantidad de ejercicios, decide cuando abandonar y reiniciar, interactuar reiteradas veces, en fin son muchos los beneficios. Por su parte el docente encuentra en ellos una ayuda significativa, pues en muchos casos en los MECs se registra toda la actividad del estudiante.

Este tipo de ambientes computacionales centran su actividad en procesos algorítmico y heurístico, en el primero predomina el aprendizaje vía trasmisión de conocimiento y en el segundo predomina el aprendizaje empírico y por descubrimiento sin embargo una de las cualidades más destacadas de los sistemas educativos computarizados es que permiten llevar a la práctica los dos enfoques educativos que aunque son opuestos se complementan ya que cada uno de ellos privilegia algún aspecto del hecho educativo y del desarrollo cognitivo en particular.

En la práctica en este ambiente computacional pretende desarrollar los dos enfoques ya que posee un sistema de ejercitación y práctica y juegos, lenguajes y 
herramientas visuales en 3D lo que permite didácticamente enriquecer la capacidad del medio ambiente educativo, incitando la actividad intelectual en términos de activar los esquemas de asimilación del estudiante, centrándolo deliberadamente en lo reflexivo y privilegiando la actividad simbólica representacional, lo cual consiste en obtener conocimiento a partir de la abstracción de las transformaciones (Escobar, 1989).

En esta propuesta se eligen los conceptos relacionados la importancia biológica y las características químicas de las sustancias asociadas en los carbohidratos, debido a que estos generalmente no se abordan en la educación media, seguramente por dejar como temática a tratar en los últimos periodos o por considerarla menos significativa con respecto a otros conceptos, a pesar de ser las biomoléculas más abundantes sobre la superficie terrestre, de representan los principales componentes de tipo energéticonutricional y de hacer parte de los contenidos básicos de ciencias naturales y educación ambiental incluidos dentro de la propuesta de lineamientos curriculares como procesos químicos y biológicos, que acercan al estudiante a comprender su entorno y construir un lenguaje científico a partir de la teoría y sus vivencias (MEN, 1998).

A continuación se presenta la propuesta de taller a realizar con estudiantes de educación media, para la enseñanza didáctica del concepto carbohidrato con apoyo del MEC.

\section{PROPUESTA \\ TALLER PARA LA ENSEÑANZA DEL CONCEPTO CARBOHIDRATO}

La aplicación de la estrategia didáctica, se lleva a cabo en diferentes etapas, mostrándose en el siguiente esquema:

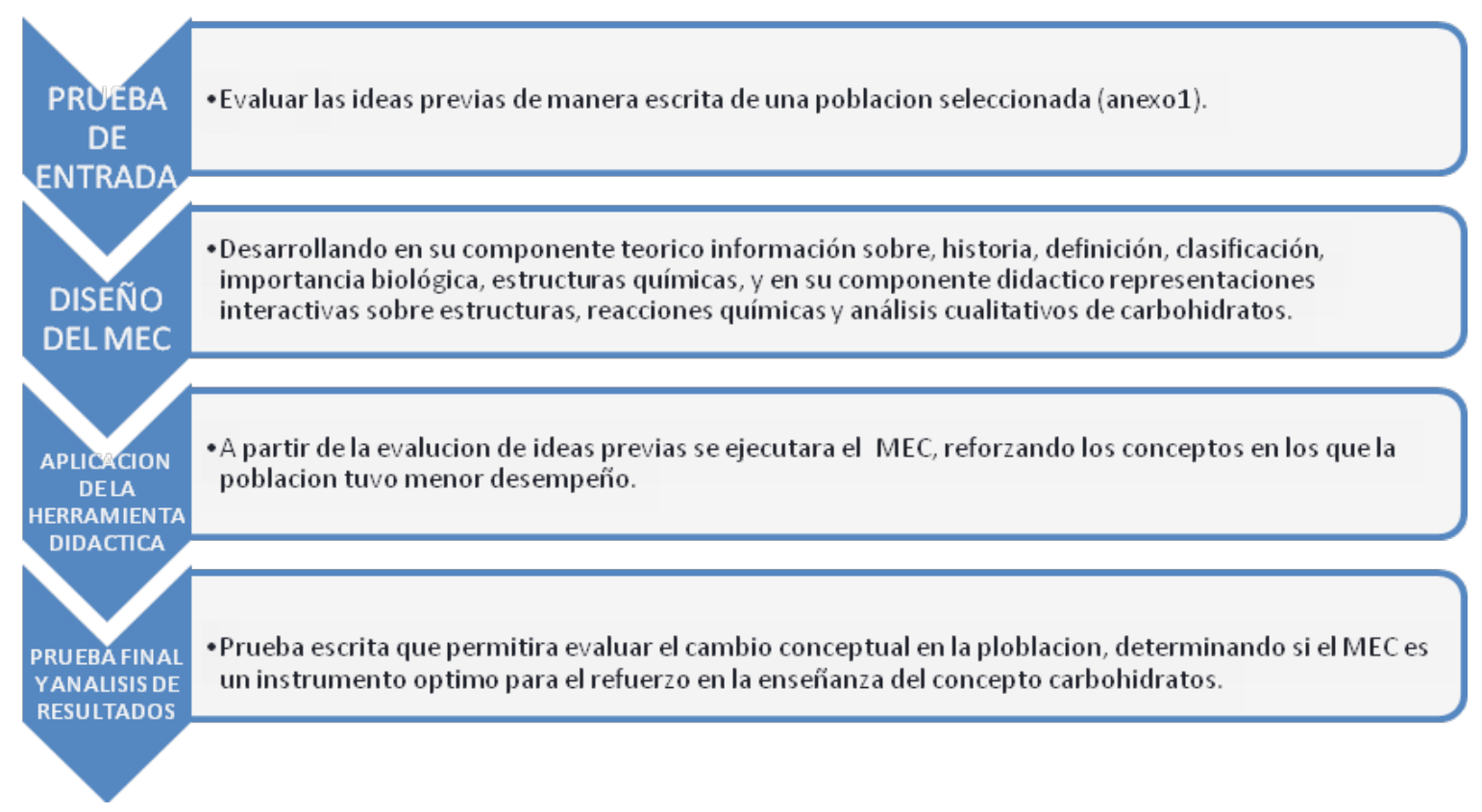

\section{Reflexiones finales}

La enseñanza de las ciencias en la educación media, se enfoca en el desarrollo de habilidades que permitan al estudiante conocer, explicar y describir fenómenos de la naturaleza, y relacionar la teoría con vivencias de su cotidianidad. Para facilitar el proceso educativo, se da la interdisciplinaridad de las ciencias naturales con la 
tecnología, buscando generar redes conceptuales que faciliten la comprensión y aprendizaje de varios conceptos fácilmente aplicables.

Es por esto que con la puesta en marcha de la presente propuesta permitirá a los estudiantes una nueva posibilidad de aprendizaje con recursos no tradicionales, aumentando su motivación y rendimiento en la temática tratada (carbohidratos). Lo cual se reflejara en los resultados satisfactorios que se obtendrán en la aplicación de la prueba final.

Podemos concebir este MEC como contenidos libres ya que facilita la articulación del aprendizaje con el desarrollo de los materiales educativos, en la medida que los MEC no sólo son construcciones colectivas fruto de un trabajo de acercamiento a la realidad de los problemas, sino que también son flexibles. Pueden ser modificados, ajustados o adaptados tantas veces como ocasiones se tenga de abordar la misma problemática por diferentes grupos de estudiantes. Así que la utilización de programas informáticos de fácil acceso y sin restricciones para la redistribución, y la adopción de los materiales construidos como contenidos libres, proporcionan a los MEC un espacio importante de apoyo al trabajo docente.

\section{Bbliografía}

Arnaz, J. (1995). La planeación curricular. México. Editorial Trillas.

Escobar, M. (1989).Ambientes computacionales y desarrollo cognitivo: perspectiva psicológica. Boletín de informática educativa. 2 (2), Proyecto SIIE, Colombia.

Galvis, A. (1988). Ambientes de enseñanza-aprendizaje enriquecidos con computador. Boletín de Informática Educativa. 1(2).

Gómez Pérez. J, (2004). Las TIC en la educación. http://boj.pntic.mec.es/jgomez46/ticedu.htm.

Ministerio de Educación Nacional. (1998). Serie lineamientos curriculares. Bogotá. 
Anexo 1

\section{MATERIAL EDUCATIVO COMPUTACIONAL (MEC) UNA HERRAMIENTA DIDÁCTICA PARA ENSEÑAR CARBOHIDRATOS PRUEBA DE ENTRADA}

\section{APRECIADO ESTUDIANTE ESTA PRUEBA ESTA DISEÑADA PARA INDAGAR SUS CONOCIMIENTOS SOBRE ALGUNOS CONCEPTOS CLAVES DE MOLECULAS ORGANICAS.}

1. La energía que utiliza el organismo para realizar cualquier acción psicomotora, es tomada de los alimentos que consumimos, que contienen diferentes sustancias supliendo necesidades vitales. ¿Cuál es esta sustancia?

a. vitaminas

b. minerales

c. carbohidratos

d. alcaloide

2. Los carbohidratos más importantes son la glucosa, el almidón y la celulosa, cada uno tiene una función específica en los organismos vivos, sin embargo leemos en revistas y escuchamos diariamente que los carbohidratos hacen subir de peso y no son necesarios para nuestro cuerpo. ¿Estás de acuerdo con esta afirmación? Justifique su respuesta.

3. Según su estructura, los carbohidratos se pueden clasificar en 3 grupos Monosacáridos, Oligosacáridos dos, Polisacáridos, esta clasificación se refiere a:

a. $\quad$ A la organización de los átomos de carbono y oxigeno.

b. $\quad$ Al número de moléculas presentes.

c. A la actividad óptica de cada cadena.

d. Al número de carbonos que contengan.

4. Las siguientes imágenes corresponden a la molécula de la glucosa. ¿En qué parámetro radica su diferencia?
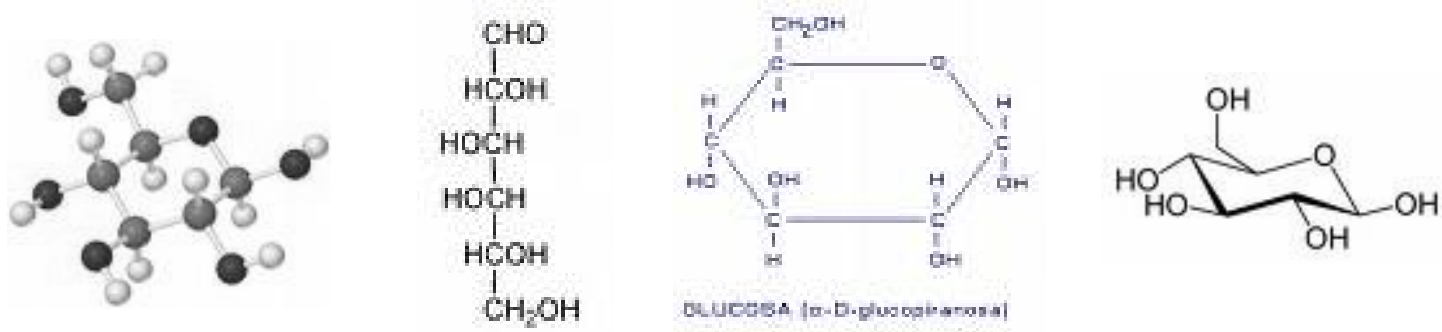

5. Los carbohidratos están presentes tanto en los alimentos de origen animal como la leche y los de origen vegeta como legumbres, cereales, harinas, verduras y frutas. ¿Cuál de los siguientes alimentos no tiene carbohidratos?

a. Huevos.

b. Uvas.

c. Repollo.

d. Ninguna de las anteriores.

\section{Responde a partir de siguiente texto las preguntas 6 y 7}

Una vez ingeridos los carbohidratos se hidrolizan a glucosa, la sustancia más simple. La glucosa es de suma importancia para el correcto funcionamiento del sistema nervioso central (SNC). Diariamente, nuestro cerebro consume más o menos 100 gr. de glucosa, cuando estamos en ayuno, el SNC recurre a los cuerpos cetónicos (Su función es suministrar energía a corazón y cerebro en ciertas situaciones excepcionales) que existen en bajas concentraciones, es por eso que en condiciones de hipoglucemia podemos sentirnos mareados o cansados.

6. La palabra hidroliza en el texto se refiere a:

a. Combinación de 2 moléculas por acción de las enzimas.

b. Reducción de la molécula a una más pequeña.

c. Desdoblamiento de la molécula de ciertos compuestos orgánicos por acción del agua. 
d. Todas las anteriores.

7. Según el texto la hipoglucemia es:

a. Un trastorno caracterizado por un descenso del nivel de glucosa en la sangre.

b. Una enfermedad que causa debilidad, temblores, excitación, ansiedad y palidez.

c. Es resultado del hiperinsulinismo, o exceso de insulina, debido a una sobredosis de insulina (en el caso de personas que sufren diabetes mellitus tipo I), o a un exceso de su producción por parte del organismo.

d. Todas las anteriores.

8. El cuerpo humano descompone o transforma la mayoría de los carbohidratos en glucosa, que es absorbida por vellosidades intestinales que las llevan al torrente sanguíneo. Conforme el nivel de la glucosa sube en la sangre, el páncreas libera una hormona que se llama insulina. La insulina es necesaria para trasladar la glucosa de la sangre a las células, donde sirve como fuente de energía. Según el texto ¿qué es la diabetes?:

a. Una enfermedad producida por una alteración del metabolismo de los carbohidratos en la que aparece una cantidad excesiva de azúcar en la sangre

b. Trastorno en el cual el páncreas no produce suficiente insulina.

c. Enfermedad metabólica caracterizada por eliminación excesiva de orina, adelgazamiento, sed intensa y otros trastornos generales.

d. Todas las anteriores

9. Los requerimientos diarios de carbohidratos son aproximadamente de $400 \mathrm{~g}$ para nuestro organismo, según esto y teniendo en cuanta que cada gramo de carbohidratos aporta una energía de 4 Kcal. ¿Qué alimentos de la tabla deberíamos tomar para cumplir con el requerimiento diario? Indique cuantos Kcal producirían.

10. Dentro del proceso escolar de que manera has abordado temas relacionados con carbohidratos. (En tu respuesta relaciona la edad, la asignatura y el grado que cursabas).

11. ¿Sabes que es un MEC material educativo computacional?, ¿has trabajo alguna temática por medio de una herramienta de tipo computacional?

"No te quejes de tu pobreza, de tu soledad o de tu suerte, enfrenta con

Valor y acepta que de una u otra manera son el resultado de tus actos y La prueba que has de ganar." 